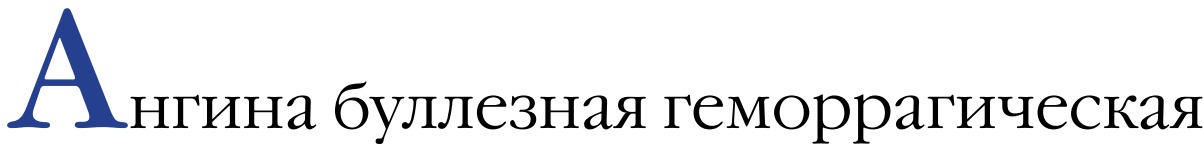

\author{
Боровиков Ю. Е.1.,
}

Ангина буллезная геморрагическая (АБГ) - термин, использующийся при описании остро возникающих субэпителиальных пузырей, заполненных кровью, на слизистой полости рта, появление которых не связано с системным заболеванием или нарушением гемостаза. В большинстве случаев для постановки диагноза достаточно характерной клинической картины, для исключения других заболеваний проводится гистологическое исследование. Мы представляем 24-летнего пациента с АБГ, протекающей с рядом клинических особенностей. Во избежание ошибок в диагностике дерматологу важно уметь распознавать АБГ.

Ключевые слова: ангина буллезная геморрагическая, пузырно-слизистый синдром, геморрагический пузырь, слизистая полости рта

Конфликт интересов: авторы заявляют об отсутствии потенциального конфликта интересов, требующего раскрытия в данной статье.

Для цитирования: Боровиков Ю. Е., Букин Ю. К. Ангина буллезная геморрагическая. Вестник дерматологии и венерологии. 2019;95(2):50-54. https://doi.org/10.25208/0042-4609-2019-95-2-50-54 


\title{
Angina bullosa hemorrhagica
}

\author{
Yuriy E. Borovikov1,*, Yuriy K. Bukin²
}

${ }^{1}$ Private Medical Practice in Dermatovenereology

Novy Val str., 18-24, Kaliningrad, 236039, Russian Federation

${ }^{2}$ Kaliningrad Regional Center of Specialized Types of Medical Care

Barnaulskaya str., 6, Kaliningrad, 236040, Russian Federation

Angina bullosa hemorrhagica $(A B H)$ is a term used to describe acute, subepithelial oral mucosal blisters filled with blood without any systemic disorder or hemostatic defect. The diagnosis of $\mathrm{ABH}$ is largely clinical, and includes elimination of other diseases at histology. We present a 24-year-old patient suffering from $\mathrm{ABH}$ with some clinical peculiarities. Recognition of $\mathrm{ABH}$ is of great importance to dermatologists, to avoid misdiagnosis.

Keywords: angina bullosa hemorrhagica, bullous vascular syndrome, hemorrhagic blister, oral mucosa

Conflict of interest: the authors state that there is no potential conflict of interest requiring disclosure in this article.

For citation: Borovikov Yu. E., Bukin Yu. K. Angina bullosa hemorrhagica. Vestnik Dermatologii i Venerologii. 2019;95(2):50-54. https://doi.org/10.25208/0042-4609-2019-95-2-50-54 
Ангина буллезная геморрагическая (angina bullosa hemorrhagica) - редкое, с доброкачественным течением заболевание, характеризующееся внезапным возникновением на слизистой ротовой полости и/или ротоглотки субэпителиальных пузырей, наполненных геморрагическим содержимым, их появление не связано с системным заболеванием или нарушением гемостаза [1].

Впервые заболевание было описано в 1933 г. как «hemophlictenosis bucal traumatica» известным аргентинским дерматологом P. L. Balina, который предположил посттравматическую природу возникновения пузырей, в большинстве случаев возникающих у пожилых пациентов, имеющих изменения в системе капилляров [2]. В разные годы в зарубежной литературе для обозначения этого состояния использовались различные термины, в частности «benign hemorrhagic bullous stomatitis», «recurrent oral hemophlyctenosis», «traumatic oral hemophlyctenosis», « localized oral purpura» [3, 4]. Термин «angina bullosa hemorrhagica» был введен N. J. Badham в 1967 г. $[1,4]$. Ссылаясь на некорректность использования термина «angina», G. Kirtschig и R. Happle в 1994 г. предложили новое название данного заболевания «stomatopompholyx hemorrhagica» [5]. Тем не менее в настоящее время в зарубежных медицинских публикациях термин «angina bullosa hemorrhagica» сохраняет свою актуальность и является наиболее часто употребляемым [1, 3, 4].

В 1972 г. А.Л. Машкиллейсоном и соавт. было описано заболевание, названное ими пузырно-сосудистым синдромом, которое характеризовалось появлением геморрагических пузырей, возникающих после травмы слизистой полости рта, у лиц с сердечно-сосудистой патологией. Авторы полагали, что сосудистая патология приводит к нарушению прочности соединения эпителия и соединительной ткани [6]. Описанная клиническая и гистологическая картина заболевания полностью соответствовала таковым при ангине буллезной геморрагической (АБГ). В последующих отечественных статьях и монографриях по заболеваниям слизистой полости рта термин «пузырно-сосудистый синдром» по-прежнему сохраняет свою актуальность и рассматривается как одно из возможных проявлений кардиологических заболеваний на слизистой полости рта [7-9].

Ангина буллезная геморрагическая (АБГ) считается редким заболеванием полости рта и ротоглотки, хотя реальная заболеваемость остается неизвестной. Есть мнение, что большинство случаев АБГ не диагностируется в связи с тем, что пациенты не обращаются за медицинской помощью из-за малосимптомности и быстрого саморазрешения высыпаний [4]. Заболевание чаще встречается у лиц среднего возраста и пожилых, преимущественно в возрастной группе старше 50 лет. Случаи АБГ не выявлялись у детей младше 10 лет. Мужчины и женщины болеют одинаково часто [1].

АБГ относится к идиопатическим заболеваниям. Конституциональная предрасположенность к АБГ может обуславливать недостаточно прочную связь между эпителием и собственно слизистой оболочкой, а также слабую фриксацию сосудов слизистой [10]. В патогенезе заболевания, вероятно, играют роль незначительные травмы слизистой при еде, употреблении горячих напитков, стоматологических процедурах [11]. Возможным этиологическим фактором является применение в лечении бронхиальной астмы ингаляторов с глюкокортикоидами, которые вызывают атрофию и уменьшение числа эластических волокон в подслизистом слое, что может приводить к разрыву капилляров и формированию пузырей при АБГ [12]. S. I. Hosain и соавт. сообщили о случае послеоперационной АБГ, вызванной интубацией и экстубацией [13]. В качестве потенциальных этиологических факторов АБГ в ряде статей называются сахарный диабет и артериальная гипертония [3, 4, 14]. Однако причинную взаимосвязь между АБГ и сахарным диабетом, а также между АБГ и гипертонической болезнью достаточно сложно установить, поскольку эти соматические заболевания достаточно часто встречаются у лиц среднего и пожилого возраста, т.е. в возрастной группе, наиболее подверженной АБГ [15]. АБГ среди других членов семьи выявлялась в $24 \%$ случаев заболевания [16]. P. Stephenson и соавт. не удалось установить никаких предрасполагающих фракторов среди 30 пациентов с АБГ в $47 \%$ случаев [17].

Характерным морфологическим элементом сыпи при АБГ является напряженный пузырь с геморрагическим содержимым. Формирование пузыря происходит стремительно, в течение нескольких секунд. У большинства пациентов заболевание представлено солитарным элементом, в 30 \% случаев - множественными пузырями. Диаметр пузырей составляет от 0,3 до 4 см. Излюбленная локализация элементов сыпи мягкое небо, реже латеральные и вентральная поверхности языка, слизистая щек, губы, десны, т.е. участки слизистой, выстланные неороговевающим эпителием [4]. Самопроизвольное вскрытие пузыря происходит в период от нескольких минут до нескольких часов с момента его возникновения с последующим образованием эрозии, которая эпителизируется в среднем течение 3-10 дней без образования рубца [14]. Элементы сыпи могут сопровождаться болевыми ощущениями [15]. Общее состояние пациента не страдает. Крайне редко пузыри при АБГ могут служить причиной развития обструкции дыхательных путей $[13,18]$. АБГ рецидивирует у $30 \%$ больных с появлением пузырей на прежнем или новом месте [14]. Частота рецидивов различна и составляет в среднем 1-2 раза в год. Заболевание имеет благоприятный прогноз [4].

В большинстве случаев для постановки диагноза АБГ достаточно характерной клинической картины и анамнеза [1]. Вместе с этим круг заболеваний с развитием пузырей на слизистой полости рта достаточно широк. Геморрагические пузыри могут возникать при лейкозах, васкулитах, тромбоцитопении и ряде других гематологических заболеваний. В связи с этим при обнаружении геморрагических пузырей необходимо проведение лабораторного исследования периферической крови и системы гемостаза $[1,3]$. Диффреренциальный диагноз АБГ проводится также с пузырчаткой, пемфигоидом слизистых оболочек, буллезным пемфигоидом, герпетиформным дерматитом, приобретенным буллезным эпидермолизом, IgA-линейным дерматозом, буллезным красным плоским лишаем, амилоидозом, фриксированной эритемой, буллезной многоформной экссудативной эритемой, буллезной системной красной волчанкой $[1,3,4,19]$. При гистологическом исследовании при АБГ выявляется геморрагический субэпителиальный пузырь в сочетании с хроническим воспалительным инфильтратом в пределах lamina propria [3]. По результатам биопсии вскрывшегося пузыря 
определяется гистологическая картина неспецифической язвы [4]. Результаты реакции прямой иммунофлюоресценции (РПИФ), направленной на выявление отложения $\lg \mathrm{A}, \lg \mathrm{G}, \lg \mathrm{M}$, С3-фрракции комплемента, фрибрина, обычно негативные [1, 3, 10, 15], в ряде случаев при РПИФ может наблюдаться фиксация вдоль базальной мембраны IgG и С3-фракции комплемента (обычно результаты трактуются как сомнительная реакция) [4, 18]. В сыворотке пациентов с АБГ при постановке реакции непрямой иммунофлюоресценции аутоантитела не выявляются [3]. Биопсия и иммунофрлюоресцентная микроскопия могут быть полезными для исключения других буллезных заболеваний [20].

Пациента, страдающего АБГ, необходимо успокоить, объяснив доброкачественность течения заболевания. Лечение АБГ симптоматическое. Для профилактики вторичной инфекции рекомендуются полоскания с раствором хлоргексидина биглюконата $[3,11]$. В редких случаях требуется назначение обезболивающих, противовоспалительных средств, топических стероидов [4]. При возникновении большого интактного пузыря и риска его дальнейшего роста его необходимо вскрыть для профрилактики обструкции дыхательных путей [15].

Рекомендуется установить длительное наблюдение за пациентами с АБГ для окончательного исключения других заболеваний, характеризующихся появлением геморрагических пузырей на слизистой полости рта и/или ротоглотки [1].

Ниже мы хотим представить собственное клиническое наблюдение.

Больной Д., 24 лет, житель Германии, обратился за помощью 12.03.18 через 1 час после возникновения на слизистой языка пузыря с кровянистым содержимым. Пузырь был обнаружен им через несколько минут после приема пищи. Появление высыпания сопровождалось умеренной болью и жжением в очаге. Пациент отрицал фракт травмы слизистой полости рта и не принимал лекарственные препараты накануне и в день возникновения сыпи. В анамнезе - атопический дерматит, контагиозный моллюск. Соматический и семейный анамнез не отягощены.

При дерматологическом осмотре (рис. 1) на левой латеральной поверхности слизистой языка определялся единичный напряженный пузырь бобовидной формы 1,3×0,6 см с геморрагическим содержимым. Высыпания на других участках слизистой и кожи отсутствовали.

Пациенту было рекомендовано дополнительное лабораторное обследование: клинический анализ крови, гемостазиограмма.

Через несколько часов пузырь начал вскрываться с выделением кровянистого содержимого. Полное опорожнение пузыря произошло к концу первых суток с момента его возникновения (рис. 2). Для профрилактики вторичной инфекции больному были назначены полоскания полости рта с 0,05 \% раствором хлоргексидина биглюконата. По результатам клинического анализа крови, гемостазиограммы патологии выявлено не было. Биопсия пузыря не проводилась в связи с быстрым самопроизвольным вскрытием элемента. С учетом клиники, характера течения заболевания и результатов лабораторного исследования был выставлен клинический диагноз ангины буллезной геморрагической. Пациент был успокоен информацией о доброкачественной природе его заболевания и благоприятном прогнозе. Пол-

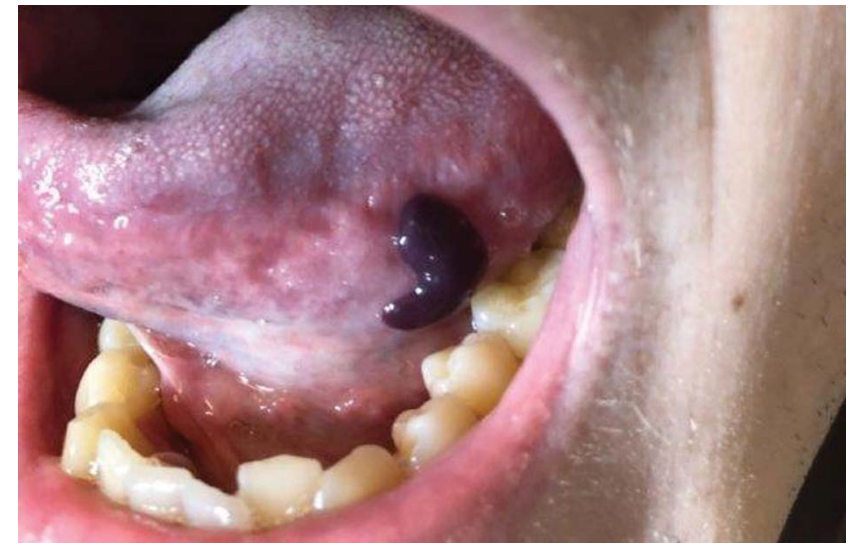

Рис. 1. Напряженный геморрагический пузырь на левой латеральной поверхности слизистой языка в дебюте заболевания

Fig. 1. Tense hemorrhagic blister on the left lateral surface of the mucous membrane of the tongue in the debut of the disease

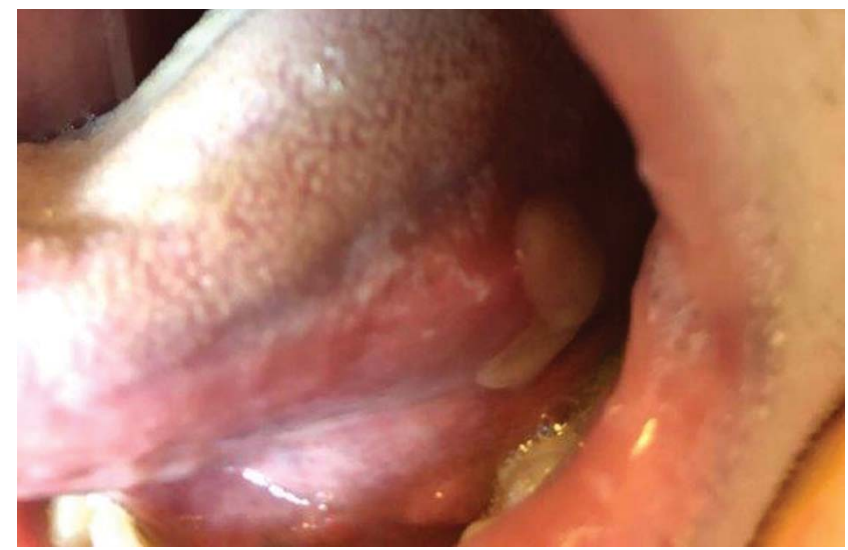

Рис. 2. Вскрывшийся пузырь к концу первых суток с момента его возникновения Fig. 2. Opened blister by the end of the first day since its inception

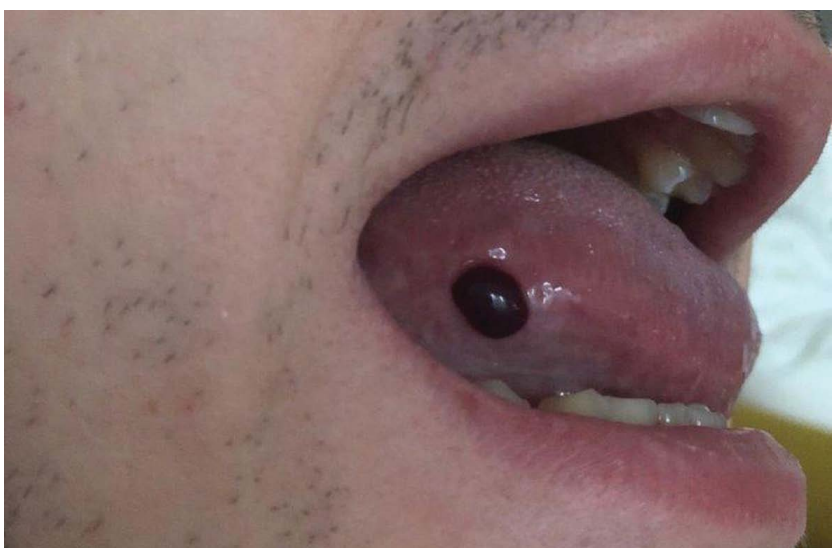

Рис. 3. Пузырь с геморрагическим содержимым на слизистой латеральной поверхности языка справа, возникший при рецидиве заболевания Fig. 3. Hemorrhagic blister on the mucous membrane of the right lateral surface of the tongue having appeared during the disease recurrence

ная эпителизация без образования рубца наступила к 14-му дню заболевания. До момента заживления пациента беспокоили локальные боль и жжение, которые затрудняли прием пищи. Пациент был взят под дальнейшее динамическое наблюдение. 
Рецидив заболевания был отмечен 08.06.18 и характеризовался появлением напряженного пузыря диаметром 0,7 см с геморрагическим содержимым на слизистой латеральной поверхности языка справа (рис. 3). Возникновение пузыря через несколько минут после приема пищи сопровождалось незначительной локальной болью. При гистологическом исследовании интактного элемента был выявлен субэпителиальный пузырь, заполненный эритроцитами и нитями фрибрина, с подлежащим воспалительным инфильтратом, состоящим преимущественно из мононуклеаров. Результат РПИФ (исследован биоптат внешне неизмененного участка слизистой в соседней с очагом зоне) для выявления фиксации IgA, IgG, IgM, С3-фрракции комплемента отрицательный. Таким образом, диагноз АБГ у данного пациента был подтвержден данными гистологического исследования и иммунофлюоресцентной микроскопии. После взятия биопсии на слизистой полости рта образовались дефекты темно-красного цвета, которые полностью эпителизировались в течение нескольких дней без образования рубца. В настоящее время мы продолжаем наблюдение за пациентом.

На наш взгляд, описанный случай представляет интерес не только в связи с редкостью данного заболевания, но и рядом особенностей возникновения и течения АБГ у данного пациента: молодой возраст, отсутствие соматического отягощения, относительно длительный период эпителизации эрозии с сохраняющимися болью и жжением на протяжении 14 дней в дебюте заболевания, рецидивирующий характер заболевания с изменением локализации пузырей.

Во избежание терминологической путаницы мы предлагаем использовать для обозначения данного заболевания общепринятое международное название - «ангина буллезная геморрагическая».

Мы рекомендуем учитывать АБГ в повседневной дерматологической практике при проведении дифференциальной диагностики буллезных дерматозов на слизистой полости рта.

\section{Литература/References}

1. Singh D., Misra N., Agrawal S., Misra P. Angina bullosa hemorrhagica. BMJ Case Rep. 2013. D0l: 10.1136/bcr-2012-008505

2. Balina P. L. Hemophlictenosis bucal traumatica. Rev Arg Dermatol. 1933:17:194-196.

3. Paci K., Varman K. M., Sayed C. J. Hemorrhagic bullae of the oral mucosa. JAAD Case Reports. 2016 Nov;2(6):433-435.

4. Alberdi-Navarro J., Gainza-Cirauqui M. L., Prieto-Elias M., AguirreUrizar J. M. Angina bullosa hemorrhagica an enigmatic oral disease. World J Stomatol. 2015;4(1):1-7.

5. Kirtschig G., Happle R. Stomatopompholyx hemorrhagica. JAAD. 1994;31(5):804-805.

6. Скрипкин Ю. К. (ред.) Кожные и венерические болезни. Т. 3. М.: Медицина, 1996. [Skripkin Yu. K. (ed.) Skin and venereal diseases. Vol. 3. Moscow: Meditsina, 1996. (In Russ.)]

7. Данилевский Н. Ф., Леонтьев В. К., Несин А. Ф., Рахний Ж. И. Заболевания слизистой оболочки полости рта. М.: ОАО «Стоматология», 2001. 271 c. [Danilevskij N. F., Leont'ev V. K., Nesin A. F., Rakhnij Zh. I. Diseases of oral mucosa. Moscow: Stomatologiya, 2001. 271 p. (In Russ.)]

8. Боровский Е. В., Машкиллейсон А. Л. Заболевания слизистой полости рта и губ. М.: МЕДпресс, 2001. 320 с. [Borovskij Е. V., Mashkillejson A. L. Diseases of oral mucosa and lips. Moscow: MEDpress, 2001. 320 p. (In Russ.)]

9. Глебова Л. И., Терехов Г. А., Фирфраров К. В. Пузырно-сосудистый синдром в практике врача стоматолога и терапевта. Стоматологпрактик. 2015;1:67-68. [Glebova L. I., Terekhov G. A., Phirpharov K. V. Bullous vascular syndrome in the practice of a therapist and a dentist. Stomatolog-praktik. 2015;1:67-68. (In Russ.)]
10. Serra D., Schonenberger de Oliveira H., Reis J. P., Vieira R., Figueiredo A. Angina bullosa hemorrhagica: a disorder to keep in mind. EJD. 2010;20(4):509-510.

11. Molano P. E., Luna S., Vasquez M. A. Angina bullosa hemorrhagica: a case report. Rev Fac Odontol Univ Antioq. 2017;28(2):422-432.

12. Punniyakodi R., Gurumani C., Thangaraj B. Angina bullosa hemorrhagica: report of two cases. International Journal of Research in Dermatology. 2017;3(1):155-157.

13. Hosain S. I., Bounds G., Stanford J. Angina bullosa hemorrhagica causing respiratory obstruction postoperatively. Anaesthesia. 1991;46:422.

14. Shashikumar B. M., Reddy R. R., Harish M. R. Oral hemorrhagic blister: An enigma. Indian J Dermatol. 2013;58:407.

15. Yayli S., Yayli A. Y. Angina bullosa hemorrhagica. JDDG. 2012;10(6):436-437.

16. Edwards S., Wilkinson J. D., Wojnarowska F. Angina bullosa hemorrhagica - a report of three cases and review of literature. Clinical and Experimental Dermatology. 1990;15:422-424.

17. Stephenson P., Lamey P-J., Scully C., Prime S. S. Angina bullosa hemorrhagica: clinical and laboratory features in 30 patients. Oral Surgery Oral Medicine Oral Pathology. 1987;63:560-565.

18. Pahl C., Yarrow S., Stevenson N., Saeed N. R., Dyar O. Angina bullosa hemorrhagica presenting as acute upper airway obstruction. British Journal of Anaesthesia. 2004:92(2):283-286.

19. Beguerie J. R., Gonzalez S. Angina bullosa hemorrhagica: Report of 11 cases. Dermatol Reports. 2014;6(1):5282.

20. Rai S., Kaur M., Goel S. Angina bullosa hemorrhagica: Report of two cases. Indian J Dermatol. 2012;57(6):503.

\section{Информация об авторах}

Юрий Евгеньевич Боровиков* — врач-дерматовенеролог частной медицинской практики по дерматовенерологии; e-mail: bory70@list.ru Юрий Константинович Букин - главный врач ГБУЗ «Центр специализированных видов медицинской помощи»

\section{Information about the authors}

Yuriy E. Borovikov* — Clinician, Dermatovenerology Doctor, Private Medical Practice in Dermatovenereology; email: bory70@list.ru Yuriy K. Bukin - Chief Doctor, Kaliningrad Regional Center of Specialized Types of Medical Care 